\title{
$Q$-analog of Determinant of a Kind of Binomial Coefficient Matrices
}

\author{
Maixue LiU And Zhizheng ZhanG*
}

We would like to thank the reviewers for their valuable suggestions.

\begin{abstract}
Recently, Ratliff and Rush gave the explanation of a kind of determinant whose entries are zero and either binomial coefficients of their negative. Such work can be considered in some generalized Pascal matrices. The purpose of this paper is to establish a $q$-analog of the result of Ratliff and Rush. Also, a $q$-analogs about problem 269 in [5] are given.
\end{abstract}

\section{INTRODUCTION}

Properties of certain determinants and their evaluation play an important role in enumerative combinatorics, and a thorough understanding of them has led to some combinatorial information. For example, in [10, 12, 13], certain computations in commutative algebra lead to matrices whose entries are binomial coefficients and in $[1,14,15]$, the determinants from Gaussian binomial coefficients have the important significance for studying plane partitions. Recently, Ratliff and Rush [11] gave the computation of a kind of determinant from binomial coefficient matrices. Such works can be considered in some generalized Pascal matrices of $[2,3,16,17,18]$. The purpose of this paper is to establish a $q$-analog of the results of Ratliff and Rush [11].

In this paper, section 2 states our main results and their interesting corollaries. In section 3, some preliminary results are obtained; and in section 4 the proof of main results are given.

2000 Mathematics Subject Classification. Primary 05A10, 11B65, 11C20; Secondary 15A15, $15 \mathrm{~A} 36$.

Key words and phrases. Gaussian binomial coefficient, matrix, determinant, Vandermonde determinant, upper triangular matrix.

${ }^{*}$ This research is supported by the National Natural Science Foundation of China (Grant No. 10471016) and the Natural Science Foundation of the Education Department of Henan Province of China (Grant No.200510482001). 


\section{The Main Results}

To state our result, we introduce some standard notation (see [6]):

$$
\begin{gathered}
{[k]=\frac{1-q^{k}}{1-q},} \\
{[k] !=[k][k-1] \cdots[1], \quad([0] !=1),} \\
{\left[\begin{array}{l}
n \\
k
\end{array}\right]=\frac{[n] !}{[k] ![n-k] !} .}
\end{gathered}
$$

Let $s \geqslant 2, n \geqslant 2$ be integers and let $m=s+n$. Let $A=A(n, s ; q)$ be the $s \times m$ cyclic matrix with the first row being

$$
(-\left[\begin{array}{l}
n \\
0
\end{array}\right],\left[\begin{array}{l}
n \\
1
\end{array}\right],-\left[\begin{array}{l}
n \\
2
\end{array}\right] q, \ldots,(-1)^{n+1}\left[\begin{array}{l}
n \\
n
\end{array}\right] q^{\frac{1}{2} n(n-1)}, \overbrace{0, \ldots, 0}^{s-1}) .
$$

By $A_{i}=A_{i}(n, s ; q)$ we denote the $i$-th row of $A$, that is

$$
A_{i}=(\overbrace{0, \ldots, 0}^{i-1},-\left[\begin{array}{l}
n \\
0
\end{array}\right],\left[\begin{array}{l}
n \\
1
\end{array}\right],-\left[\begin{array}{l}
n \\
2
\end{array}\right] q, \ldots,(-1)^{n+1}\left[\begin{array}{l}
n \\
n
\end{array}\right] q^{\frac{1}{2} n(n-1)}, \overbrace{0, \ldots, 0}^{s-i}) .
$$

Write

$$
A_{i}^{+}=A_{i}^{+}(n, s ; q)=(\overbrace{0, \ldots, 0}^{i-1},\left[\begin{array}{l}
n \\
0
\end{array}\right],\left[\begin{array}{l}
n \\
1
\end{array}\right],\left[\begin{array}{l}
n \\
2
\end{array}\right] q, \ldots,\left[\begin{array}{l}
n \\
n
\end{array}\right] q^{\frac{1}{2} n(n-1)}, \overbrace{0, \ldots, 0}^{s-i}) .
$$

For the selected integers $1 \leqslant r_{1}<r_{2}<\cdots<r_{n} \leqslant m$, let $M_{q}\left(n, s ; r_{1}, \ldots, r_{n}\right)$ denote the $s \times s$ matrix obtained by deleting columns $r_{1}, r_{2}, \ldots, r_{n}$ from $A(n, s ; q)$, and $M_{q}^{+}\left(n, s ; r_{1}, r_{2}, \ldots, r_{n}\right)$ the matrix of absolute values of the entries of $M_{q}\left(n, s ; r_{1}, r_{2}, \ldots, r_{n}\right)$.

The following Theorem 2.1 is a $q$-analog of Theorem 1.1. in [1].

\section{Theorem 2.1.}

(i) $\operatorname{det}\left(M_{q}\left(n, s ; r_{1}, r_{2}, \ldots, r_{n}\right)\right)=(-1)^{y+s} q^{\frac{1}{2} n(n-1)(n+s+1)} \prod_{1 \leqslant i<j \leqslant n} \frac{q^{-r_{i}}-q^{-r_{j}}}{q^{j}-q^{i}}$, where $y=n s+n(n+1) / 2+\sum_{i=1}^{n} r_{i}$;

(ii) $\operatorname{det}\left(M_{q}^{+}\left(n, s ; r_{1}, r_{2}, \ldots, r_{n}\right)\right)=(-1)^{y+s} \operatorname{det}\left(M_{q}\left(n, s ; r_{1}, r_{2}, \ldots, r_{n}\right)\right)$.

By taking some special values of $r_{i}$ in Theorem 2.1 we get the following interesting corollaries.

Corollary 2.1.1. Let $g$ be a positive integer such that $r_{n}+g \leqslant m$. Then

$$
\begin{aligned}
\operatorname{det}\left(M _ { q } \left(n, s ; r_{1}+g, r_{2}+g, \ldots\right.\right. & \left.\left., r_{n}+g\right)\right)= \\
& =(-1)^{n g} q^{-\frac{1}{2} n(n-1) g} \operatorname{det}\left(M_{q}\left(n, s ; r_{1}, r_{2}, \ldots, r_{n}\right)\right)
\end{aligned}
$$


and

$\operatorname{det}\left(M_{q}^{+}\left(n, s ; r_{1}+g, r_{2}+g, \ldots, r_{n}+g\right)\right)=q^{-\frac{1}{2} n(n-1) g} \operatorname{det}\left(M_{q}^{+}\left(n, s ; r_{1}, r_{2}, \ldots, r_{n}\right)\right)$.

Corollary 2.1.2. Let $a \geqslant 1, b \geqslant 1, n \geqslant 2$ be integers and choose integer s such that $a+(n-1) b \leqslant n+s=m$. Then

$$
\begin{aligned}
& \operatorname{det}\left(M_{q}(n, s ; a, a+b, a+2 b, \ldots, a+(n-1) b)\right)= \\
= & (-1)^{y+s} q^{\frac{1}{2} n(n-1)((1-b) n+s-a+1)} \prod_{1 \leqslant i<j \leqslant n} \frac{q^{b j}-q^{b i}}{q^{j}-q^{i}},
\end{aligned}
$$

where $y=n(s+a)+\frac{1}{2} n((1+b) n-b+1)$;

$$
\begin{aligned}
& \operatorname{det}\left(M_{q}^{+}(n, s ; a, a+b, a+2 b, \ldots, a+(n-1) b)\right)= \\
= & q^{\frac{1}{2} n(n-1)((1-b) n+s-a+1)} \prod_{1 \leqslant i<j \leqslant n} \frac{q^{b j}-q^{b i}}{q^{j}-q^{i}} .
\end{aligned}
$$

Corollary 2.1.3.

$$
\operatorname{det}\left(M_{q}(n, s ; 1,2,3, \ldots, n)\right)=(-1)^{s(n+1)} q^{\frac{1}{2} n(n-1) s},
$$

and

$$
\operatorname{det}\left(M_{q}^{+}(n, s ; 1,2,3, \ldots, n)\right)=q^{\frac{1}{2} n(n-1) s} .
$$

The following Theorem 2.2 is a $q$-analog of Theorem 2.1 in [11].

\section{Theorem 2.2.}

$$
\operatorname{det}\left(\left[\begin{array}{c}
r_{i} \\
j-1
\end{array}\right]\right)_{i, j=1,2, \ldots, n}=q^{\frac{1}{2} n(n-1)} \prod_{1 \leqslant i<j \leqslant n} \frac{q^{r_{j}}-q^{r_{i}}}{q^{j}-q^{i}}
$$

Let $Q$ be the doubly infinite matrices whose $k$-th rows are

$$
\left(\left[\begin{array}{l}
k \\
0
\end{array}\right],\left[\begin{array}{l}
k \\
1
\end{array}\right],\left[\begin{array}{l}
k \\
2
\end{array}\right],\left[\begin{array}{l}
k \\
3
\end{array}\right], \ldots\right)
$$

where $\left[\begin{array}{l}k \\ j\end{array}\right]=0$ if $j>k$. Thus the nonzero entries yield the justified form of Gauss-Pascal triangles. Let $Q\left(r_{1}, r_{2}, \ldots, r_{n}\right)$ be the $n \times n$ submatrix of $Q$ formed by the first $n$ columns and the rows $r_{1}, r_{2}, \ldots, r_{n}$ of $Q$. The matrices in Theorems 2.2 is $Q\left(r_{1}, r_{2}, \ldots, r_{n}\right)$. When $r \rightarrow 1$, it becomes the formula that is given without proof in [5] as the problem 269.

Corollary 2.2.1.

$$
\operatorname{det}\left(\left[\begin{array}{c}
m-r_{i} \\
n-j
\end{array}\right]\right)_{i, j=1,2, \ldots, n}=q^{\frac{1}{2} n(n-1)(m+1)} \prod_{1 \leqslant i<j \leqslant n} \frac{q^{-r_{i}}-q^{-r_{j}}}{q^{j}-q^{i}} .
$$




\section{Lemmas}

In this section we first give a simple result which allows us to replace the matrix $M_{q}\left(n, s ; r_{1}, r_{2}, \ldots, r_{n}\right)$ with another one which has a simpler structure, at the expense of increasing the size of the matrix.

Lemma 3.1. Let $e_{i}$ be the row vector with 1 in the $i$-th place and zeros elsewhere. Let $B_{q}\left(n, s ; r_{1}, r_{2}, \ldots, r_{n}\right)$ be the $m \times m$ matrix obtained from $A(n, s ; q)$ by adjoining the rows $e_{r_{1}}, e_{r_{2}}, \ldots, e_{r_{n}}$ to the bottom, namely

$$
B_{q}\left(n, s ; r_{1}, r_{2}, \ldots, r_{n}\right)=\left(\begin{array}{c}
A(n, s ; q) \\
e_{r_{1}} \\
e_{r_{2}} \\
\vdots \\
e_{r_{n}}
\end{array}\right),
$$

and

$$
B_{q}^{+}\left(n, s ; r_{1}, r_{2}, \ldots, r_{n}\right)=\left(\begin{array}{c}
A^{+}(n, s ; q) \\
e_{r_{1}} \\
e_{r_{2}} \\
\vdots \\
e_{r_{n}}
\end{array}\right)
$$

Write $y=n s+\frac{1}{2} n(n+1)+\sum_{i=1}^{n} r_{1}$. Then

$$
\begin{aligned}
\operatorname{det}\left(M_{q}\left(n, s ; r_{1}, r_{2}, \ldots, r_{n}\right)\right) & =(-1)^{y} \operatorname{det}\left(B_{q}\left(n, s ; r_{1}, r_{2}, \ldots, r_{n}\right)\right), \\
\operatorname{det}\left(M_{q}^{+}\left(n, s ; r_{1}, r_{2}, \ldots, r_{n}\right)\right) & =(-1)^{y} \operatorname{det}\left(B_{q}^{+}\left(n, s ; r_{1}, r_{2}, \ldots, r_{n}\right)\right) .
\end{aligned}
$$

The statement and proof of this lemma are similar to Lemma 2.1. of paper [11]. Here we state no longer its proof.

Suppose $e_{i j}$ is the matrix which is zero except for the entry 1 in the $i j$ place. For $k \geqslant 2$, let

$$
T_{k}(q)=\operatorname{diag}\left(q^{-1}, q^{-2}, \ldots, q^{-k}\right)\left(I_{k}+q^{-1} e_{1,2}\right)\left(I_{k}+q^{-1} e_{2,3}\right) \cdots\left(I_{k}+q^{-1} e_{k-1, k}\right),
$$

and

$$
S_{k}(q)=\operatorname{diag}\left(q^{-1}, q^{-2}, \ldots, q^{-k}\right)\left(I_{k}-q^{-1} e_{1,2}\right)\left(I_{k}-q^{-1} e_{2,3}\right) \cdots\left(I_{k}-q^{-1} e_{k-1, k}\right)
$$

namely,

$$
T_{k}(q)=\left(\begin{array}{ccccc}
q^{-1} & q^{-2} & q^{-3} & \cdots & q^{-k} \\
0 & q^{-2} & q^{-3} & \cdots & q^{-k} \\
0 & 0 & q^{-3} & \cdots & q^{-k} \\
\vdots & \vdots & \vdots & \ddots & \vdots \\
0 & 0 & 0 & \cdots & q^{-k}
\end{array}\right)
$$


and

$$
S_{k}(q)=\left(\begin{array}{ccccc}
q^{-1} & -q^{-2} & q^{-3} & \cdots & (-1)^{1+k} q^{-k} \\
0 & q^{-2} & -q^{-3} & \cdots & (-1)^{2+k} q^{-k} \\
0 & 0 & q^{-3} & \cdots & (-1)^{3+k} q^{-k} \\
\vdots & \vdots & \vdots & \ddots & \vdots \\
0 & 0 & 0 & \cdots & (-1)^{k+k} q^{-k}
\end{array}\right)
$$

For $0 \leqslant u<m$, let

$$
\begin{aligned}
& T_{m, u}(q)=\left(\begin{array}{cc}
T_{m-u}(q) & 0 \\
0 & \dot{I}_{u}
\end{array}\right), \\
& S_{m, u}(q)=\left(\begin{array}{cc}
S_{m-u}(q) & 0 \\
0 & I_{u}
\end{array}\right) .
\end{aligned}
$$

Then, $T_{k, 0}(q)=T_{k}(q)$ and $S_{k, 0}(q)=S_{k}(q)$.

Now we consider how $T_{m, u}(q)$ and $S_{m, u}(q)$ operate on a row of $B_{q}\left(n, s ; r_{1}, r_{2}, \ldots, r_{n}\right)$ or $B_{q}^{+}\left(n, s ; r_{1}, r_{2}, \ldots, r_{n}\right)$ :

Lemma 3.2. (i) Let $A_{i}(n, s ; q)$ be the $i$-th row of $A$, as denoted in (2.1). Then for $0 \leqslant u \leqslant m-(i+n)$ we have

$$
A_{i}(n, s ; q) T_{m, u}=q^{-i} A_{i}(n-1, s+1 ; q) .
$$

(ii) Let $A_{i}^{+}(n, s ; q)$ be the row vector in (2.2). Then for $0 \leqslant u \leqslant m-(i+n)$ we have

$$
A_{i}^{+}(n, s ; q) S_{m, u}=q^{-i} A_{i}^{+}(n-1, s+1 ; q) .
$$

Proof. Both (3.3) and (3.4) follow from the identity

$$
\sum_{j=0}^{k}(-1)^{j+1}\left[\begin{array}{l}
n \\
j
\end{array}\right] q^{\frac{1}{2} j(j-1)}=(-1)^{k+1}\left[\begin{array}{c}
n-1 \\
k
\end{array}\right] q^{\frac{1}{2} k(k+1)}
$$

(see $[9])$.

Corollary 3.2.1. Let $A_{i}(n, s ; q)$ and $A_{i}^{+}(n, s ; q)$ be as in above lemma and let $0 \leqslant u \leqslant m-i-n$. Then

$$
\begin{gathered}
A_{i}(n, s ; q) T_{m, u}(q) T_{m, u+1}(q) T_{m, u+2}(q) \cdots T_{m, u+n-1}(q)=-q^{n i} e_{i} \\
A_{i}^{+}(n, s ; q) S_{m, u}(q) S_{m, u+1}(q) S_{m, u+2}(q) \cdots S_{m, u+n-1}(q)=-q^{n i} e_{i}
\end{gathered}
$$

$(i=1,2, \ldots, m-n)$.

Lemma 3.3. Let $G_{k}^{(j)}=q^{-(n-j)(k+j+i)}\left[\begin{array}{l}n-1+k \\ n-1-j\end{array}\right]$ and $H_{k}^{(j)}=(-1)^{j+k} G_{k}^{j}$. Then for $1 \leqslant i \leqslant m$ we have 
(i)

$$
\begin{aligned}
e_{i} T_{m, 0}(q) T_{m, 1}(q) \cdots T_{m, n-1}(q)= & (\overbrace{0, \ldots, 0}^{i-1}, G_{0}^{(0)}, G_{1}^{(0)}, \ldots, G_{m-n-i}^{(0)}, G_{m-n-i+1}^{(0)}, \\
& \left.G_{m-n-i+1}^{(1)}, G_{m-n-i+1}^{(2)}, \ldots, G_{m-n-i+1}^{(n-1)}\right)
\end{aligned}
$$

$$
\begin{aligned}
e_{i} S_{m, 0}(q) S_{m, 1}(q) \cdots S_{m, n-1}(q)= & (\overbrace{0, \ldots, 0}^{i-1}, H_{0}^{(0)}, H_{1}^{(0)}, \ldots, H_{m-n-i}^{(0)}, H_{m-n-i+1}^{(0)}, \\
& \left.H_{m-n-i+1}^{(1)}, H_{m-n-i+1}^{(2)}, \ldots, H_{m-n-i+1}^{(n-1)}\right)
\end{aligned}
$$

Proof. Use $\sum_{j=1}^{n} q^{(n-j)(i+1)}\left[\begin{array}{l}j \\ i\end{array}\right]=\left[\begin{array}{c}n+1 \\ i+1\end{array}\right]$ (see [7]) and induction.

\section{Lemma 3.4.}

$$
\operatorname{det}\left(\left\langle r_{i}\right\rangle_{j-1}\right)_{i, j=1,2, \ldots, n}=(-1)^{\frac{1}{2} n(n-1)} q^{-\left(\begin{array}{c}
n \\
3
\end{array}\right)} \prod_{1 \leqslant i<j \leqslant n}\left(q^{r_{j}}-q^{r_{i}}\right),
$$

where $\left\langle r_{i}\right\rangle_{j}=\prod_{k=1}^{j}\left(1-q^{r_{i}-k+1}\right)$.

Proof. Replacing $q$ by $q^{-1}$ in the identity

$$
\prod_{k=1}^{n}\left(1-t q^{k-1}\right)=\sum_{k=0}^{n}(-1)^{k} q^{\frac{1}{2} k(k-1)}\left[\begin{array}{l}
n \\
k
\end{array}\right] t^{k}
$$

(see [8]), we have

$$
\prod_{k=1}^{n}\left(1-t q^{-k+1}\right)=\sum_{k=0}^{n}(-1)^{k} q^{-\frac{1}{2} k(k-1)-k(n-k)}\left[\begin{array}{l}
n \\
k
\end{array}\right] t^{k},
$$

from which, taking $t \rightarrow q^{r_{i}}$ it follows

$$
\begin{aligned}
\left\langle r_{i}\right\rangle_{j-1} & =\prod_{k=1}^{j-1}\left(1-q^{r_{i}-k+1}\right)=\sum_{k=0}^{j-1}(-1)^{k} q^{-\frac{1}{2} k(k-1)-k(j-1-k)}\left[\begin{array}{c}
j-1 \\
k
\end{array}\right] q^{r_{i} k} \\
& =\sum_{s=1}^{j}(-1)^{s-1} q^{-\frac{1}{2}(s-1)(2 j-s-2)}\left[\begin{array}{l}
j-1 \\
s-1
\end{array}\right] q^{r_{i}(s-1} .
\end{aligned}
$$

Hence

$$
\operatorname{det}\left(\left\langle r_{i}\right\rangle_{j-1}\right)=\operatorname{det}\left((-1)^{i-1} q^{-\frac{1}{2}(i-1)(2 j-i-2)}\left[\begin{array}{l}
j-1 \\
i-1
\end{array}\right]\right) \operatorname{det}\left(q^{r_{i}(j-1)}\right) .
$$

Our result follows from that the former in the above product of two determinants is upper triangular and the latter is Vandermonde determinant. 


\section{The Proofs of Theorems}

Proof of Theorem 2.2.

$$
\begin{aligned}
& \operatorname{det}\left(\left[\begin{array}{c}
r_{i} \\
j-1
\end{array}\right]\right)_{i, j=1,2, \ldots, n}= \\
= & \prod_{k=1}^{n} \frac{1}{\langle k-1\rangle_{k-1}} \operatorname{det}\left(\left\langle r_{i}\right\rangle_{j-1}\right)_{i, j=1,2, \ldots, n}= \\
= & (-1)^{\frac{1}{2} n(n-1} q^{\frac{1}{6} n(n-1)(n+1)} \prod_{1 \leqslant i<j \leqslant n} \frac{1}{q^{j}-q^{i}} \operatorname{det}\left(\left\langle r_{i}\right\rangle_{j-1}\right)_{i, j=1,2, \ldots, n} .
\end{aligned}
$$

Applying Lemma 3.4 we can get Theorem 2.2.

Proof of Corollary 2.3.1.

$$
\begin{aligned}
& \operatorname{det}\left(\left[\begin{array}{c}
m-r_{i} \\
n-j
\end{array}\right]\right)_{i, j=1,2, \ldots, n}=\quad \text { (Reversing the order of rows and columns) } \\
& =\operatorname{det}\left(\left[\begin{array}{c}
m-r_{n-i+1} \\
j-1
\end{array}\right]\right)_{i, j=1,2, \ldots, n} \quad\left(\text { Taking } m-r_{n-i+1}=p_{i}, i=1,2, \ldots, n\right) \\
& =\operatorname{det}\left(\left[\begin{array}{c}
p_{i} \\
j-1
\end{array}\right]\right)_{i, j=1,2, \ldots, n}=(\text { Applying theorem 2.2) } \\
& =q^{\frac{1}{2} n(n-1)} \prod_{1 \leqslant i<j \leqslant n} \frac{q^{p_{j}}-q^{p_{i}}}{q^{j}-q^{i}}=\left(\text { Taking } p_{i}=m-r_{n-i+1}\right) \\
& =q^{\frac{1}{2} n(n-1)} \prod_{1 \leqslant i<j \leqslant n} \frac{q^{m-r_{n-j+1}}-q^{m-r_{n-i+1}}}{q^{j}-q^{i}}= \\
& =q^{\frac{1}{2} n(n-1)(m+1)} \prod_{1 \leqslant i<j \leqslant n} \frac{q^{-r_{n-j+1}}-q^{-r_{n-i+1}}}{q^{j}-q^{i}}= \\
& =q^{\frac{1}{2} n(n-1)(m+1)} \prod_{1 \leqslant i<j \leqslant n} \frac{q^{-r_{i}}-q^{-r_{j}}}{q^{j}-q^{i}} .
\end{aligned}
$$

Proof of Theorem 2.1. Let

$$
J(q)=\left(\begin{array}{cccc}
q^{-n} & & & \\
& q^{-2 n} & & \\
& & \ddots & \\
& & & q^{-s n}
\end{array}\right) .
$$

From corollary 3.2.1 we have

$$
A(n, s ; q) T_{m, 0}(q) T_{m, 1}(q) \cdots T_{m, n-1}(q)=(-J(q), 0),
$$


and

$$
A^{+}(n, s ; q) S_{m, 0}(q) S_{m, 1}(q) \cdots S_{m, n-1}(q)=(J(q), 0)
$$

Then

$$
\begin{aligned}
& B_{q}\left(n, s ; r_{1}, r_{2}, \ldots, r_{n}\right) T_{m, 0}(q) T_{m, 1}(q) \cdots T_{m, n-1}(q)= \\
= & \left(\begin{array}{c}
A(n, s ; q) T_{m, 0}(q) T_{m, 1}(q) \cdots T_{m, n-1}(q) \\
e_{r_{1}} T_{m, 0}(q) T_{m, 1}(q) \cdots T_{m, n-1}(q) \\
\vdots \\
e_{r_{n}} T_{m, 0}(q) T_{m, 1}(q) \cdots T_{m, n-1}(q)
\end{array}\right)=\left(\begin{array}{cc}
-J(q) & 0 \\
* & X
\end{array}\right),
\end{aligned}
$$

and

$$
\begin{aligned}
& B_{q}^{+}\left(n, s ; r_{1}, r_{2}, \ldots, r_{n}\right) S_{m, 0}(q) S_{m, 1}(q) \cdots S_{m, n-1}(q)= \\
= & \left(\begin{array}{c}
A^{+}(n, s ; q) S_{m, 0}(q) S_{m, 1}(q) \cdots S_{m, n-1}(q) \\
e_{r_{1}} S_{m, 0}(q) S_{m, 1}(q) \cdots S_{m, n-1}(q) \\
\vdots \\
e_{r_{n}} S_{m, 0}(q) S_{m, 1}(q) \cdots S_{m, n-1}(q)
\end{array}\right)=\left(\begin{array}{cc}
J(q) & 0 \\
* & Y
\end{array}\right) .
\end{aligned}
$$

It is easily seen that for each $u$,

$$
\begin{gathered}
\operatorname{det}\left(T_{m, u}(q)\right)=\operatorname{det}\left(T_{m-u}(q)\right)=q^{-1-2-\cdots-(m-u)}=q^{-\frac{1}{2}(m-u)(m-u+1)}, \\
\operatorname{det}\left(S_{m, u}(q)\right)=\operatorname{det}\left(S_{m-u}(q)\right)=q^{-\frac{1}{2}(m-u)(m-u+1)}
\end{gathered}
$$

and

$$
\operatorname{det}\left(\begin{array}{cc}
-J(q) & 0 \\
* & X
\end{array}\right)=\operatorname{det}(-J(q)) \operatorname{det}(X)
$$

and

$$
\operatorname{det}\left(\begin{array}{cc}
J(q) & 0 \\
* & Y
\end{array}\right)=\operatorname{det}(J(q)) \operatorname{det}(Y) .
$$

We thus have

$$
\begin{aligned}
& \operatorname{det}\left(B_{q}\left(n, s ; r_{1}, r_{2}, \ldots, r_{n}\right)\right)= \\
= & q^{\frac{1}{2} \sum_{u=0}^{n-1}(m-u)(m-u+1)}(-1)^{s} q^{-2-2 n-\cdots-s n} \operatorname{det}(X)= \\
= & (-1)^{s} q^{\frac{1}{2} \sum_{u=0}^{n-1}(m-u)(m-u+1)-\frac{1}{2} n s(s+1)} \operatorname{det}(X)= \\
= & (-1)^{s} q^{\frac{1}{2}(m+1)(m+2) n-\frac{1}{4} n(n+1)(2 m+3)+\frac{1}{12} n(n+1)(2 n+1)-\frac{1}{2} n s(s+1)} \operatorname{det}(X)= \\
= & (-1)^{s} q^{\frac{1}{6} n(n-1)(n+1)+\frac{1}{2} n(n+1)(s+1)} \operatorname{det}(X) .
\end{aligned}
$$

and

$$
\operatorname{det}\left(B_{q}^{+}\left(n, s ; r_{1}, r_{2}, \ldots, r_{n}\right)\right)=q^{\frac{1}{6} n(n-1)(n+1)+\frac{1}{2} n(n+1)(s+1)} \operatorname{det}(Y) .
$$

We now proceed to compute $\operatorname{det} X$ and $\operatorname{det} Y$.

By Lemma 3.3 the last $n$ entries of $e_{r_{i}} T_{m, 0}(q) T_{m, 1}(q) \cdots T_{m, n-1}(q)$ are

$$
\left(G_{m-n-r_{i}+1}^{(0)}, G_{m-n-r_{i}+1}^{(1)}, \ldots, G_{m-n-r_{i}+1}^{(n-1)}\right) .
$$


Thus

$$
\begin{aligned}
\operatorname{det} X & =\operatorname{det}\left(G_{m-n-r_{i}+1}^{(j-1)}\right)=\operatorname{det}\left(q^{-(n-j+1)(m-n+1)}\left[\begin{array}{c}
m-r_{i} \\
n-j
\end{array}\right]\right)_{i, j=1,2,3, \ldots, n}= \\
& =q^{-\sum_{j=1}^{n}(n-j+1)(m-n+j)} \operatorname{det}\left(\left[\begin{array}{c}
m-r_{i} \\
n-j
\end{array}\right]\right)_{i, j=1,2, \ldots, n}= \\
& =q^{-\frac{1}{6} n(n+1)(n+2)-\frac{1}{2} n(n+1) s} \operatorname{det}\left(\left[\begin{array}{c}
m-r_{i} \\
n-j
\end{array}\right]\right)_{i, j=1,2, \ldots, n} .
\end{aligned}
$$

Similar to above methods, by Lemma 3.3, the last $n$ entries of $e_{r_{1}} S_{m, 0}(q) S_{m, 1}(q) \cdots S_{m, n-1}(q)$ are

$$
\left(H_{m-n-r_{i}+1}^{(0)}, H_{m-n-r_{i}+1}^{(1)}, \ldots, H_{m-n-r_{i}+1}^{(n-1)}\right) .
$$

Thus

$$
\begin{aligned}
\operatorname{det} Y & =\operatorname{det}\left(H_{m-n-r_{i}+1}^{(j-1)}\right)_{i, j=1,2,3, \ldots, n}= \\
& =\operatorname{det}\left((-1)^{m-n+j-r_{i}} q^{-(n-j+1)(m-n+j)}\left[\begin{array}{c}
m-r_{i} \\
n-j
\end{array}\right]\right)_{i, j=1,2, \ldots, n}= \\
& =(-1)^{y^{\prime}} q^{-\frac{1}{6} n(n+1)(n+2)-\frac{1}{2} n(n+1) s} \operatorname{det}\left(\left[\begin{array}{c}
m-r_{i} \\
n-j
\end{array}\right]\right)_{i, j=1,2, \ldots, n} .
\end{aligned}
$$

where $y^{\prime}=\frac{1}{2} n(n+1)+n s-\sum_{i=1}^{n} r_{i}$.

Comparing (4.7) and (4.8), we have $\operatorname{det} X=(-1)^{y^{\prime}} \operatorname{det} Y$, and comparing (4.5) and (4.6), we have

$$
\operatorname{det}\left(B_{q}\left(n, s ; r_{1}, r_{2}, \ldots, r_{n}\right)\right)=(-1)^{s+y^{\prime}} \operatorname{det}\left(B_{q}^{+}\left(n, s ; r_{1}, r_{2}, \ldots, r_{n}\right)\right) .
$$

It follows from Lemma 3.1 that

$$
\operatorname{det}\left(M_{q}^{+}\left(n, s ; r_{1}, r_{2}, \ldots, r_{n}\right)\right)=(-1)^{s+y^{\prime}} \operatorname{det}\left(M_{q}\left(n, s ; r_{1}, r_{2}, \ldots, r_{n}\right)\right) .
$$

Note that the number $y$ defined in Theorem 2.1 has the same parity with $y^{\prime}$ :

$$
y^{\prime}+y=-\frac{1}{2} n(n+1)+n s-\sum_{i=1}^{n} r_{i}+n s+\frac{n(n+1)}{2}+\sum_{i=1}^{n} r_{i} \equiv 0 \quad(\bmod 2) .
$$

It follows the second formula in the theorem. The first is obtained from (4.5) and (4.7) and Lemma 3.1.

The proof is completed.

\section{REFERENCES}

[1] G.E. Anderws, The Theory of Partitions, Addison-Wesley, Reading, MA, 1976.

[2] R. Brawer and M. Pirovino, The linear algebra of the Pascal matrix, Linear Algebra App. 174(1992) 13-23

[3] G.S. Call and D.J. Vellman, Pascal's matrices, Amer. Math. Monthly 100(1993) 372-376

[4] L. Comtet, Advanced Combinatorics, D. Reidel Publishing Company, 1974. 
[5] D.K. Faddeev and I.S. Slominskii, Problems in Higher Algebra, Freeman, San Francisco, 1965.

[6] W.P. Johnson, q-Extension of identities of Abel-Rothe type, Discrete Math. 159(1996) 161177

[7] J. Konvalina, Generalized binomial coefficients and the subset-subspace problem, Advanced of Applied Math., 21(1998) 228-240

[8] A. Mercier, Identities containing Gaussian binomial coefficients, Deiscrete Math. 76(1989) $67-73$

[9] A. Mercier, Quelques identites pour les q-analogues, Utilitas Mathematica 38(1990) 33-41

[10] T.T. Moh, On the unboundedness of generators of prime ideals in power series rings of three variables, J. Math. Soc. Jpn. 26(1974) 722-731

[11] L.J. Ratliff Jr and D.E. Rush, Triangular powers of integers from determinants of binomial coefficients, Linear Algebra App. 291(1999) 125-142.

[12] P. Roberts, A computation of local cohomology, in Comutative algebra: Syzygies, multiplicities, and birational algebra, Contemp. Math. 159(1994) 351-356

[13] A.K. Singh, A computation of tight closure in diagonal hypersurfaces, J. Algebra 203(1998) $579-589$

[14] R.P. Stanley, Theory and application of plane partitions I, Studies in Appl. Math., 50.2(1971) 167-188

[15] R.P. Stanley, Theory and application of plane partitions II, Studies in Appl. Math. $\mathbf{5 0 . 3}(1971) 51-60$

[16] Z. Zhang, The linear algebra of the generalized Pascal matrix, Linear Algebra App. 250(1997) 51-60

[17] Z. Zhang and M. Liu, An extension of the linear algebra of the generalized Pascal matrix, Linear Algebra App. 271(1998) 169-177

[18] Z. Zhang and T. Wang, Generalized Pascal matrix and recurrence sequences, Linear Algebra App. 283(1998) 289-299

Department of Mathematics
Luoyang Teacher's College
Luoyang
Henan 471022
P.R. China

Department of Mathematics

LuOyang Teacher's College

LUOYANG,

HENAN 471022

P.R. China

E-mail address: zhzhzhang-yang@163.com 